\title{
A retrospective study of surgically treated subperiosteal orbital abscesses derived from acute bacterial ethmoid
} sinusitis*

\author{
J.H. Therchilsen', A.A. Muhamad², A. Bilde', C. von Buchwald' \\ ' Department of Otolaryngology, Head \& Neck Surgery and Audiology, Copenhagen University Hospital, Copenhagen, Denmark \\ 2 Department of Neuroradiology, Copenhagen University Hospital, Copenhagen, Denmark
}

Rhinology Online, Vol 2: 103 - 110, 2019

http://doi.org/10.4193/RHINOL/19.021

*Received for publication:

May 29, 2019

Accepted: October 20, 2019

Published: October 30, 2019

\begin{abstract}
Background: Subperiosteal abscesses (SPOA) are a complication of acute purulent sinusitis, and their optimal treatment regime is a controversial topic. Some otolaryngologists consider surgery to be mandatory in the treatment of subperiosteal abscesses others advocate that especially paediatric patients can be treated medically in some cases. The purpose of this study was to evaluate our current treatment guidelines for subperiosteal abscesses.
\end{abstract}

Methodology: A retrospective study was undertaken from 2010 to 2016, including patients registered with the diagnosis of subperiosteal abscesses derived from acute sinusitis based on clinical diagnoses, and CTs or MRIs. All patients received treatment with IV-antibiotics and were booked for prompt surgery. The main outcome measures were CT-measurements and intraoperatively identified pus. Treatment included antibiotic choice, microbiology, and outcomes.

Results: Thirty-one patients were included in the study having the diagnosis of sinusitis and SPOA. Per operatively, we found pus in the subperiosteal space in 21/31 patients and 17 of the patients with available contrast-enhanced CT scans. When patients were grouped based on radiographically confirmed abscesses or not per the preoperative MRI or CT, we found that the presumed abscess volume was $0.83 \mathrm{~mL}$ greater in the patients, who had an abscess confirmed during surgery. Two patients needed endoscopic re-operation. In addition, three patients had sequela at the last follow-up, two with minor subjective sequelae due to oedema most likely resolving themselves over time and one with memory and concentration difficulties due to intracranial empyema.

Conclusion: Endoscopic drainage of SPOA combined with an intravenous antibiotic is a safe and effective treatment strategy when executed in trained surgical hands, which is substantiated by low recurrence and morbidity rates. Because of the relatively high incidence of contrast-enhanced CT-confirmed subperiosteal abscesses that cannot be identified intraoperatively, we suggest considering other parameters such as volume of the abscess to determine whether surgical intervention is needed.

Key words: subperiosteal orbital abscess, sinusitis complications, acute sinusitis, ESS, FESS

\section{Introduction}

A subperiosteal orbital abscess (SPOA) arises from acute sinusitis in particularly acute ethmoiditis where the inflammation spreads through vascular channels or directly through bony de- hiscence in lamina papyracea ${ }^{(1,2)}$. It is a disease capable of rapid progression, and both intracranial and orbital extensions can have vital consequences if not treated promptly and adequately. SPOA, as a complication of acute bacterial rhinosinusitis (ABRS), 
possesses a threat if it is left untreated. It might result in blindness, meningitis, cerebral abscess, and death ${ }^{(3-5)}$. Acute viral rhinosinusitis (ARS) is a common occurrence with a prevalence of 6 cases or $15 \%$. Between $0.5-2.0 \%$ of virus-induced acute rhinosinusitis, cases are complicated by a bacterial infection and of these cases, 3-11\% develop intracranial, osseous, or orbital complications ${ }^{(6,7)}$.

In 1970 Chandler et al. ${ }^{(3)}$ described and classified orbital complications derived from ARS (Table 1). This classifications system remains the preferred analytic tool for assessing the severity of orbital complications and ensures homogeneity in both research and clinical study. Chandler et al. ${ }^{(3)}$ define an SPOA as a collection of pus between the periorbita and the bony wall of the orbit.

The diagnosis SPOA can be based both on clinical findings and/ or on a contrast-enhanced computed tomography (CECT) or MRI scan. The clinical factors that determine this diagnosis are the deterioration/impairment of extraocular movement, proptosis, periorbital oedema, diplopia, and vision loss ${ }^{(6)}$. The CECT scan typically shows a hypodense area encapsulated by a thin periosteal contrast-enhanced convex membrane, juxtaposed to a sinus ${ }^{(1,8)}$.

Both a typical CECT-scan and the clinical presentation of a SPOA are illustrated in one of our patients with a medially placed SPOA (Figure 1 and 2).

There is general agreement regarding the pathogenesis and diagnosis in the literature, but when it comes to treatment, more controversy exists about which treatment strategy to choose. Some authors forcefully argue that prompt surgery is the correct clinical approach for CECT-confirmed SPOA ${ }^{(9-12)}$. Others advocate an initial use of antibiotics and that surgery only should be used if certain criteria are fulfilled ${ }^{(5,13-15)}$. Most articles that are more inclined to surgery in all cases of SPOA are earlier studies ${ }^{(9-11)}$, and some authors, still writing on this topic, have changed their approaches to a more conservative one ${ }^{(10,16,17)}$.

In recent years there has been a trend towards not perceiving SPOA as a homogeneous condition but rather dependent on age ${ }^{(1,16-18)}$. This perception is fuelled by bacteriological studies of SPOA cultures, demonstrating a predominance of simple monomicrobial infections with no anaerobic microbes in children under the age of nine ${ }^{(16,18)}$. This discovery might be the reason why recent studies have successfully treated medially placed SPOAs in children with an initial course of antibiotics if the child did not show symptoms of advanced SPOA ${ }^{(1,4,15-17)}$.

There is a growing body of evidence supporting a conservative approach in children with SPOAs. The general recommendation of the European Position Paper on Rhinosinusitis (EPOS) is still
Table 1. Chandler's classification of orbital complications of sinusitis.

\begin{tabular}{cl}
\hline Stage & \multicolumn{1}{c}{ Description } \\
\hline I & Inflammatory edema (preseptal cellulitis) \\
\hline II & Orbital cellulitis (postseptal cellulitis) \\
\hline III & Subperiosteal abscess \\
IV & Orbital abscess \\
V & Cavernous sinus thrombosis \\
\hline
\end{tabular}

that SPOAs should be treated with surgery and adjuvant antibiotics ${ }^{(6)}$.

This approach is also the recommended clinical practice at the Department of Otolaryngology, Head \& Neck Surgery, Copenhagen University Hospital, Copenhagen, Denmark.

The present article aims to evaluate our treatment of sinogenic SPOAs at the Department of Otolaryngology, Head \& Neck Surgery, Copenhagen University Hospital, Copenhagen, Denmark, in comparison to the literature.

\section{Materials and methods}

\section{Study design}

A retrospective, clinical study was undertaken of all cases that had been admitted at the Department of Otolaryngology, Head \& Neck Surgery, Copenhagen University Hospital, Copenhagen, Denmark, on an emergency basis, diagnosed with acute sinogenic orbital complications and treated with surgery between January 1, 2010, until January 1 2016. The study protocol was approved by The Danish Data Protection Agency.

\section{Data collection}

The patient charts were identified by searching for the ICD10 diagnosis of acute sinusitis (DJ010-14 + DJ019) among all patients admitted to our department. Our department acts as a tertiary referral centre which serves as the sole Otolaryngology Department for approximately 1.8 million citizens. Besides, a search in the surgical records was conducted, and all patients who had undergone endoscopic sinus surgery were identified, and their charts examined to identify patients treated for acute sinusitis.

Patients were included in the study if their charts documented evidence of SPOA derived from acute ethmoiditis and if a CT or MRI confirmed sinogenic orbital complication. We chose to include only patients when a close relationship between the abscess and opacified ethmoid sinus was present to align the data, results, and conclusions with the classical presentation of SPOA. Patients were excluded if they had a history of facial trauma, craniosynostosis, immunodeficiency, or malignancy. The patients were diagnosed using the Chandler classification (Table 1). Patient charts were evaluated for age, gender, and result of 


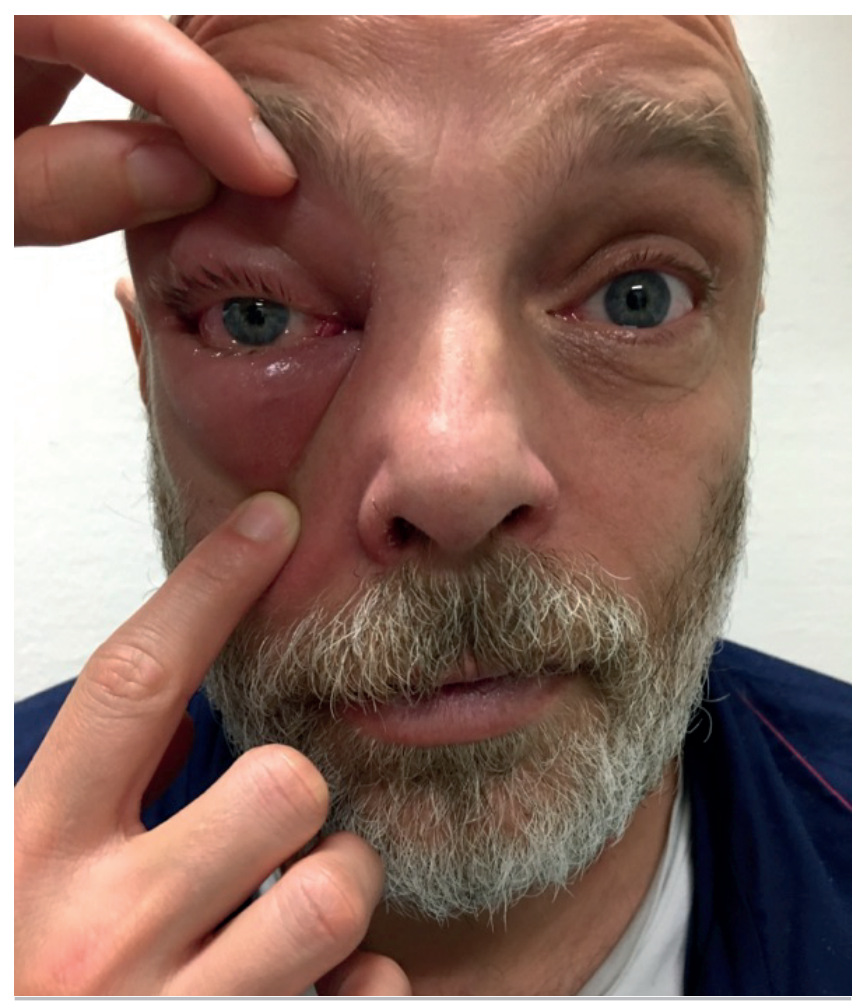

Figure 1. Preoperative photo of a patient treated at our department for a medially placed SPOA.

ophthalmology examination, if available. The ophthalmology examination included evaluation of visual acuity, colour vision, diplopia, ocular motility, periorbital oedema, chemosis, proptosis, and intraocular pressure. It was not possible in all cases to perform a full examination either due to young age, pain and/or severe periorbital oedemas.

All patients underwent a CT or MRI scan before surgery to identify the SPOA and to plan the surgical approach. The scans and the descriptions were reviewed, and the measurements of the SPOA, as well as the location, were identified by a neuroradiologist. Measurement of the SPOA was only recognised as valid in data analysis if a CECT scan was available. The volume of the abscess was calculated using the formula for calculating the volume of an ellipsoid (4/3. $\pi \cdot a b c)$, which best correlates with the abscess shape ${ }^{(19,20)}$.

Endoscopic surgery was, in all cases, performed using computerassisted endoscopic sinus surgery (CAS-ESS). The endoscopic drainage was executed under general anaesthesia using uncinectomy, anterior and posterior ethmoidectomy, and penetration of lamina papyracea. The presence of subperiosteal orbital pus was noted. If other sinuses on the affected side were opacified, drainage was secured. After the drainage was secured, the nasal cavity was irrigated and left without any packing to secure drainage. Postoperative instructions were to use saline irrigation 3-4 times a day.

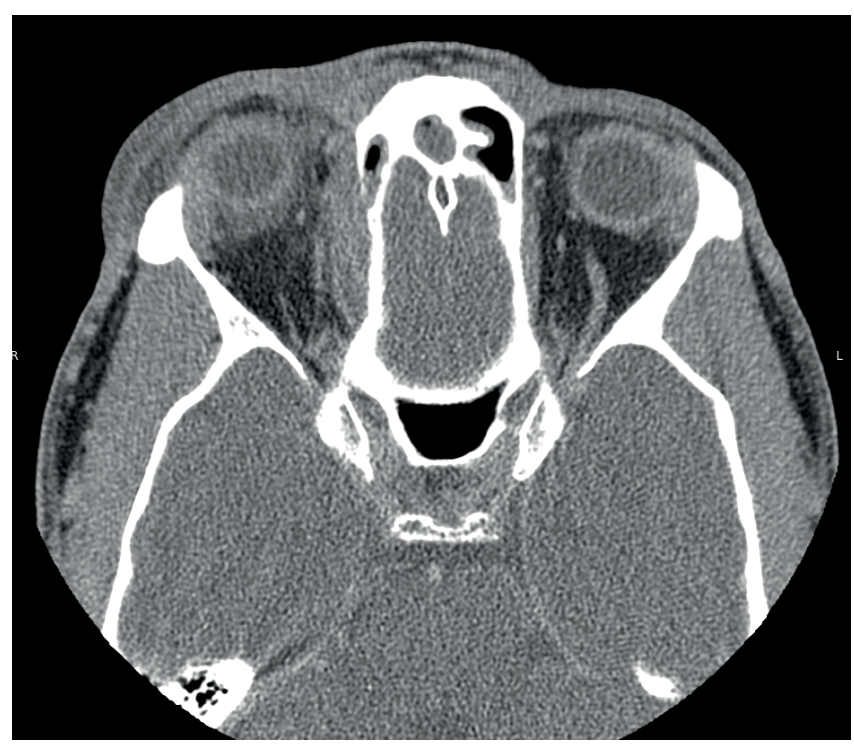

Figure 2. Axial view of a CECT scan of the patient in Figure 1.

Culture growth, clinical course, length of stay at our department, and the antibiotics used were identified.

Recurrence defined as reoperation and sequelae/morbidity was evaluated using electronically updated patient charts.

Physical examination results were only included if performed by the ophthalmologic service.

\section{Statistical analysis}

All data were entered into an Excel spreadsheet (Microsoft Corp., Redmond, WA, USA). The data analysis was performed using IMB SPSS Statistics 23.0 software (Statistical Package for Social Sciences, IBM Corp. in Armonk, NY, USA). Independent t-tests were used to compare numeric values. All tests were two-sided and performed at a $5 \%$ significance level $(p<5 \%)$ with $95 \%$ confidence intervals.

\section{Results}

A total of 31 patients were included in the study. The mean age was 14.5 years (range $1.1-57.8$ years). The patient age distribution was positively skewed within the paediatric segment $(n=23)$ with $15 / 23(65.2 \%)$ patients under the age of 9 . Across the entire age group, a bimodal distribution was found. Thirteen (41.9\%) were males, and 18 (58.1\%) were females.

All 31 patients underwent surgery with adjuvant intravenous antibiotic treatment. The recommended IV-antibiotic regime per our guideline is Cefuroxim and Metronidazol. All patients received IV-antibiotics before and after surgery. The length of antibiotic treatment varied according to physician evaluation of symptom remission. All patients received subsequent peroral antibiotic treatment. Of the 31 patients diagnosed with SPOA, 21 patients (67.7\%) were identified with pus intraoperatively in the subperiosteal orbital space. In 9/31 (29.0\%) patients, there 
Table 2. Mean dimensions of subperiosteal abscesses in the 24 CECT scanned patients measured from preoperative scans.

\begin{tabular}{|lccc|}
\hline & \multicolumn{2}{c}{$\begin{array}{c}\text { Intraoperative SPOA } \\
\text { pus identification }\end{array}$} & \\
& Yes & No & $\begin{array}{c}\text { Statistical } \\
\text { significance }\end{array}$ \\
\hline Anterior-posterior $(\mathrm{mm})$ & 27.3 & 24.3 & 0.200 \\
\hline Side-to-side $(\mathrm{mm})$ & 8,2 & 6,7 & 0.127 \\
\hline Cranio-caudal $(\mathrm{mm})$ & 16.6 & 14.2 & 0.089 \\
\hline Volume $(\mathrm{mL})$ & 1.98 & 1.15 & 0.047 \\
\hline
\end{tabular}

Table 3. Microbiology culture results from the 31 patients obtained during surgery.

\begin{tabular}{|lc|}
\hline Non-haemolytic streptococcus Anginosus Gp & Number (\%) \\
\hline Staphylococcus Aureus & $8(25.8 \%)$ \\
\hline Haemophilus Influenzae & $6(19.4 \%)$ \\
\hline Haemolytic Streptococcus Gp A & $5(16.1 \%)$ \\
\hline Streptococcus Pneumonia & $4(12.95)$ \\
\hline Prevotella Oris & $3(9.7 \%)$ \\
\hline Positive growth & $1(3.2 \%)$ \\
\hline No pathogenic growth & $1(3.2 \%)$ \\
\hline
\end{tabular}

Table 4. Overview of the initial antibiotic treatment of the 31 patients.

\begin{tabular}{|lc|}
\hline & Number (\%) \\
\hline Cefuroxim & $24(77.4)$ \\
\hline Metronidazol & $14(45.2)$ \\
\hline Ciprofloxacin & $2(6.5)$ \\
\hline Meropenem & $2(6.5)$ \\
\hline Benzylpenicillin & $2(6.5)$ \\
\hline Ampicillin & $2(6.5)$ \\
\hline Dalacin & $1(3.2)$ \\
\hline Fusidin & $1(3.2)$ \\
\hline Vancomycin & $1(3.2)$ \\
\hline Polytherapy & $20(64.5)$ \\
\hline Monotherapy & $14(35.5)$ \\
\hline Cefuroxim + Metronidazol & $13(38.7)$ \\
\hline Monotherapy - Cefuroxim & $10(32.3)$ \\
\hline Ceftriaxon & $1(3.2)$ \\
\hline
\end{tabular}

was no evidence of pus, and in one case out of 31 (3.2\%) the chart did not clarify whether there was pus evident.

The 31 patients underwent a total of 34 surgeries (1.1 mean, range 1-2). In all 31 cases, a CAS-ESS approach was used, and in two cases the surgery was combined with external drainage via an anterior orbitotomy because the abscess in these cases was located superiorly in the orbit.

Abscesses were primarily located in the medial or superior orbit in respective 26/31 (83.9 \%) patients and 3/31 (9.7\%) patients. In 2/31 (6.5\%) patients, the abscesses were disseminated to be present both in the medial and superior orbit in one patient and medial and inferior orbit in the other patient. The mean age for solitary medial abscess placement was 14.0 years, and for other placements the mean age was 17.15 years.

Average time from CT confirmed SPOA until surgery was less than 24 hours for 22/31 (71\%). Seven out of 31 (22\%) patients underwent surgery the subsequent day. One (3\%) patient had surgery performed on day three after submission because the initial CECT scan showed no SPOA. However, on day three, the patient developed diplopia, and an SPOA was identified on the second CT-scan, and the patient was scheduled for surgery. The recurrence was examined using electronically updated charts at minimum one-month post-surgery, giving a mean follow-up duration of 880.3 days, and no patients had a recurrent SPOA. Thirty-one patients underwent endoscopic surgery, and three patients needed endoscopic re-operation with two cases considered to be surgical failures giving a surgical failure rate of $6.5 \%$. A 9-year-old girl presented with meningeal enhancement, osteomyelitis of margo supraorbitalis, and a superior SPOA. She had endoscopic surgery and an anterior orbitotomy performed in joint venture with an ophthalmic surgeon. Five days later she underwent a planned sinoscopi under general anaesthesia intended to present a sinus clearance and is therefore not considered surgical failure. At her last follow-up, 16 days after the surgery, she had persistent ptosis and slight restriction of ocular motility. An 8-year-old boy with a medial abscess treated with endoscopic surgery was re-operated 5 days post-surgery due to abscess reformation and decreasing visual acuity. Lastly a 14-year-old boy was endoscopically operated on, but surgery had to stop before the putative abscess was reached due to difficulty in obtaining haemostatic control in ethmoidal region. However, the subsequent clinical course was without complications. No patients needed a third surgery.

Twenty-one of the 31 (67.7\%) patients had an examination performed by the ophthalmologic service before surgery, and the remaining 10 (32.2\%) patients had an ophthalmologic examination performed solely by an otolaryngologist. Visual impairment comprised of visual acuity and colour vision was present in 8/21 (38.1\%). Ten out of 21 (47.6\%) patients had diplopia. Restricted ocular motility was diagnosed in 14/21 (66.7\%) patients. Proptosis was present in 16/21 (76.2\%) patients. Chemosis was present in 4/21 (19\%) patients. Increased intraocular pressure was found in 8/21 (38.1\%) patients. All patients had periorbital oedema. Digitally stored contrast-enhanced scans were available for 
review for 25/31 (80.6\%) patients and were reviewed by a neuroradiologist (AAM). In 17/24 (70.8\%) of the CECT scans, a subperiosteal abscess was found intraoperatively, and in one case the chart did not declare whether pus was evident in the subperiosteal space. The dimensions and volumes of the SPOAs were identified for all patients with a CECT scan and the means calculated anterior-posterior length (AP) $26.6 \mathrm{~mm}$, side-to-side length (SS) $7.8 \mathrm{~mm}$, craniocaudal (CC) $16.2 \mathrm{~mm}$, volume $1.79 \mathrm{~mL}$. We found a difference in means for the SPOA CT measurements when dividing the patients into two groups depending on intraoperative identification of subperiosteal orbital pus (Table 2). During surgery, cultures were obtained from the ethmoidal sinuses for all 31 patients and were positive in 24/31 (77.4\%). Abscess cultures from the SPOA were obtained from 17 patients, including seven with concurrent sinus cultures. Eleven patients had only sinus cultures. Five patients had additional tissue samples taken and send for microbiologic analysis. In one case, a sample from a subdural empyema was analysed.

When patients were grouped depending on the number of microorganisms found in surgical cultures, we found a 7.23 years' difference though this was not statistically significant $(p=0.41)$. The identified microorganisms are listed in Table 3.

Anaerobic organisms were present in one patient who had a subdural empyema. No Methicillin-resistant Staphylococcus aureus (MRSA) was cultured. All patients received IV antibiotics during hospitalisation (Table 4).

Three of the 31 (9.7\%) patients in this series had morbidity at the last follow-up. A 52-year-old woman presented with a medial abscess, slight vision loss, severe chemosis, exophthalmos, and experienced subjectively slightly decreased vision 12 days after surgery. The patient was referred for further follow up to a private ophthalmologist. An 18-year-old presented with subdural empyema and ann SPOA suspected to be derived from acute bacterial rhinosinusitis He underwent surgery for both subdural empyema and SPOA and had a prolonged hospital stay of 42 days due to the intracranial empyema. The third patient with morbidity is mentioned concerning reoperation. No mortalities occurred.

\section{Discussion}

We undertook a retrospective review of 31 patient charts to evaluate the management of sinogenic orbital complications at the Department of Otolaryngology, Head \& Neck Surgery and Audiology, Copenhagen University Hospital, Copenhagen, Denmark. Our study showed that the subset of patients referred to our tertiary treatment centre swiftly received surgical treatment for their SPOA when radiographically diagnosed and with a low level of recurrence and morbidity compared to other studies ${ }^{6,12)}$. Due to our surgical treatment of all CECT-confirmed SPOAs, we can present, to our knowledge, one of the largest retrospective studies of surgically treated SPOA secondary to acute sinusitis. The question whether surgical drainage is required for all SPOA patients was first put forth by Welsh and Welsh in 1974, and in later years the medical, more conservative approach has gained ground with Harris as its proponent ${ }^{(21,22)}$. Harris has advocated since the early 90 s that children under the age of nine years should be treated with an initial medical therapy if there is no compromise of vision. Harris' seminal work in bacteriologic studies of SPOAs played a pivotal role in generating the idea of an initial medical approach for young children with SPOA $(16,18,22)$. The idea is based on bacteriological data suggesting that children under nine years of age experience more simple infections, more compliable with medical treatment, due in part to no presence of anaerobes growth ${ }^{(18)}$. This finding has been constant through a 35-yearlong bacteriologic study of surgically treated SPOAs ${ }^{(17)}$. In the Liao and Harris' article on the bacteriology of SPOAs they point out that the bacteriology of medically treated SPOAs is unknown ${ }^{(17)}$. Since we operate all CT confirmed SPOAs, we can present a complete bacteriologic description, though limited by negative culture results. In line with Liao and Harris, we found no anaerobe cultures in patients under the age of nine ${ }^{(17)}$. Like Harris, and later Brown et al., we found a trend towards an increasing number of pathogens with increasing age suggesting more complex infections with increasing age ${ }^{(17,23)}$. The most common bacteriologic yields from the surgical cultures in our study were NHS Anginosus gp (milleri gp) and Staphylococcus aureus. Similar results are found in two other extensive bacteriologic papers on SPOA cultures - Liao et al. and Liao and Harris ${ }^{(17,24)}$.

In contrast to other retrospective studies, we did not perceive certain skin flora as positive culture yields but disregarded them as contamination ${ }^{(4,20,25)}$. Of the six positively identified Staphylococcus aureus cultures, three were found in endoscopically obtained sinus cultures not concordant with the yield from the abscess cultures, begging the question whether the Staphylococcus aureus in these cases reflect nasal contamination. In the American literature, MRSA has become an increasing therapeutic issue ${ }^{(17,24)}$. In our study, we did no identify MRSA.

As per the guidelines of our department, intravenous Cefuroxim and Metronidazol should be administered to both treat aerobic and possible anaerobic components of the SPOA. In line with this recommendation, the most used antibiotic was Cefuroxim, and the most used polytherapy was Cefuroxim and Metronidazol. Despite this common drug usage, we found the individual considerations of the attending surgeon to have an impact on antibiotic choice. As per antibiotic choice, Harris, suggests a reasonable presumptive antibiotic therapy consists of a cephalosporin for patients under the age of nine years with an addition of Clindamycin to cover the anaerobe component possible in older patients ${ }^{(18)}$. As an alternative, he suggests Amoxicillin with Clavulanic acid. This antibiotic regime, except the recommenda- 
tion of Clindamycin, is much in line what a recently published article on orbital complications due to sinusitis recommends ${ }^{(10,12)}$. The recommended duration of IV-treatments are scarcely examined, and the duration recommendations range widely between studies (from 10 days to 3 days) ${ }^{(15,26)}$. The recommendation of a recently published article designed to answer this question reports a mean IV-treatment duration of 4 days but concludes that the clinical judgement id based on initial $\mathrm{CT}$ findings and the evolving signs, symptoms, and laboratory profile, not to be superseded by a fixed notion of mandatory IV-duration ${ }^{(10)}$.

In our study, we did not identify pus in the possible subperiosteal space during surgery in 6/24 (25\%) patients with a CECT, excluding one patient where it was not noted whether pus was evident. The positive predictive value of CECT is, therefore, 75 $\%$. This result is similar to the positive predictive value of a retrospective study by Brown et al. and it stresses the importance of other clinical characteristics in evaluating whether or not to operate ${ }^{(23)}$. Other studies suggest that the decision to perform surgery in SPOA patients cannot rely solely on a CECT description, but remains a clinical decision ${ }^{(4,25,27)}$. The challenge of fitting all our patients into Chandler's classification system maybe because it was not based on CECT findings and, therefore, radiographic documentation does not suffice in the SPOA diagnosis (28). This problem of classification has been pointed out previously because although CECT imaging suggests a likelihood of an abscess, it does not unequivocally differentiate between an abscess and a phlegmon, especially when the fluid collection is small ${ }^{(15,25,26)}$. Because a phlegmon is believed to be responsive to antibiotic treatment, ophthalmological examination and CECTmeasurements have been used to determine better appropriate surgical candidates ${ }^{(15)}$.

In our study, we found mean CECT abscess measurements of AP $26.6 \mathrm{~mm}$, SS $7.8 \mathrm{~mm}$, CC $16.2 \mathrm{~mm}$, and volume $1.79 \mathrm{~mL}$. Comparing our average $\mathrm{CT}$ measurements to the radiography operational criteria outlined in other studies, a satisfying correlation was found ${ }^{(4,19,25,29)}$. Since we performed endoscopic surgery on all patients with $\mathrm{CT}$ confirmed SPOA, we were able to perform a comparative analysis of SPOA patients. The patients were grouped, depending on whether an abscess was identified in the subperiosteal space during surgery. We found a difference in mean volume of $0.83 \mathrm{~mL}(1.97 \mathrm{~mL}-1.14 \mathrm{~mL}, \mathrm{p}=0.047)$. This finding supports the natural logic that the bigger the volume, the more likely it is to be an abscess. The reason behind the relatively large number of false-positive identifications an of SPOA using CT-imaging most likely is that it represents phlegmon inflammation ${ }^{(26)}$. In line with our study, Tabarino et al. concluded in a retrospective study of 32 patients diagnosed with SPOA that all abscesses with a volume greater than $0.5 \mathrm{~mL}$ were operated upon ${ }^{(29)}$. Gavriel et al. also concluded that abscesses with larger volumes than $0.5 \mathrm{~mL}$ should be candidates for surgical interven- tion ${ }^{(19)}$.

Consequently, we do not wish to operate when no abscess is present because medial phlegmon is believed to be susceptible to medical therapy ${ }^{(26)}$. We did not find a statistical difference in SS, CC, or AP measurements when grouped by intraoperatively identified pus in the subperiosteal space. Other studies have examined CT measurements and established a correlation between these parameters and whether to operate. Ryan et al. found that the majority of small abscesses less than $10 \mathrm{~mm}$ in SS measurement, in patients less than 6 years of age could be managed medically ${ }^{(25)}$. Oxford et al. identified a significant difference in abscess width between medically and surgically treated patients $(2.5-14 \mathrm{~mm}, \mathrm{p}<0.001)^{(4)}$.

Abscess relation in the orbit has often been spoken of both concerning surgical approach and when assessing the risk of intracranial extension. Consequently, urgent drainage of nonmedial abscesses is, therefore, recommended by several authors $(1,10,20,22,27)$. Superior and inferior abscesses are not as easily assessed by the endoscopic approach, but rather a combined approach must be considered. A simple external approach without endoscopic surgery leaves the nidus of infection behind to make a possible re-accumulation of the abscess and, thus, the need for recurrent surgery ${ }^{(30)}$.

Besides the CECT criteria to determine the medical versus surgical approach, an ophthalmologic examination is essential. Twenty-one of 31 (67.7\%) patients had such an examination performed by the ophthalmologic service before surgery. In some patient charts, it was argued that visual compromise was present, identified by the ENT surgeon, and the delay of the surgery due to further examination was ill-advised. Other charts did not clarify why an ophthalmologic examination was not ordered. Since no medically treated group is present in this study, a comparative analysis is not possible. If medical treatment is considered, the importance of ophthalmologic examination results is crucial ${ }^{(4)}$. Several studies executed in ophthalmologic departments have taken the use of such criteria to group patients in surgical or medical treatment groups ${ }^{(1,4,5,16,20,23)}$. A prospective study by Garcia and Harris proposed that surgical intervention was indicated if visual loss or afferent pupillary defect should be detected at any point ${ }^{(16)}$. Oxford et al. define a set of more rigorous criteria for medical management including normal pupil, vision, retina, no diplopia, intraocular pressure $<20 \mathrm{~mm} \mathrm{Hg}$, proptosis of $5 \mathrm{~mm}$ or less, and a width of abscess on CT of less than $4 \mathrm{~mm}{ }^{(4)}$. These criteria are postulated after a retrospective analysis and as such, must be evaluated with caution. Despite the retrospective nature of the studies on the subject of SPOAs, numerous articles agree that visual compromise is an exclusionary medical criterion ${ }^{(4,15,22,25,27)}$. If medical treatment of a subset of patients at our department at some point becomes feasible, the ophthalmologic examination with an emphasis on visual compromise would be central in the evaluation of possible 
medical treatment.

To our knowledge, the present study represents one of the largest surgically treated SPOA groups published in the literature, and because all patients were treated with endoscopic drainage it uniquely qualifies our bacteriological results as representative of SPOA. Moreover, the study can bear evidence of how many CECT-diagnosed SPOAs are verified intraoperatively to give the idea of the positive predictive value of CECT. Even though our study offers excellent insight into the effective surgical treatment of SPOA, it is restricted by the retrospective nature of the study which weakens the level of evidence. Lastly, it must be considered that our department acts as a tertiary referral centre and, as such, is only implemented in patient treatment of sinogenic orbital complications if endoscopic surgical treatment is thought necessary by the referring party. This fact might lead to institutional referral bias.

\section{Conclusions}

We found the endoscopic drainage of SPOA to be an acceptable, safe and effective treatment strategy when executed in trained surgical hands, which is substantiated by low surgical failure and morbidity rates. Even though endoscopic drainage is deemed a relatively safe method of treatment, an aspect of overtreatment must be considered if surgical drainage of all SPOA patients is chosen. We found CT measurements of the SPOA's volume to be of statistical significance in determining whether an SPOA is present.

As it is difficult to estimate the causal effects in retrospective studies, the next step should be to construct a prospective randomised study with a control group where the medical treatment of sinogenic SPOAs in a subset of patients are examined versus surgical.

\section{Acknowledgement}

We are grateful for aid with the statistical analysis by Copenhagen University's Biostatistical Unit.

\section{Authorship contribution}

Johan $\mathrm{H}$. Therchilsen, design, data mining, writing, analysis, editing; Ali A. Muhamad, data analysis; Anders Bilde, design, writing, analysis, editing; Christian von Buchwald, editing, revising.

\section{Conflict of interest}

None.

\section{Ethics approval and consent to participate}

Consent from the patient in Figure 1 and 2 was obtained in writing. Study protocol was approved by the Danish Data Protection Agency.

\section{Consent for publication}

Not applicable

\section{Availability of data and materials}

The datasets used during the current study are available from the corresponding author on reasonable request.

\section{Funding}

None

\section{References}

1. Greenberg MF, Pollard ZF. Medical treatment of pediatric subperiosteal orbital abscess secondary to sinusitis. J AAPOS 1998;2(6):351-5.

2. Jackson K, Baker SR. Clinical implications of orbital cellulitis. Laryngoscope. 1986 May;96(5):568-74.

3. Chandler JR, Langenbrunner DJ, Stevens ER. The pathogenesis of orbital complications in acute sinusitis. Vol. 80, Laryngoscope. 1970. p. 1414-28.

4. Oxford LE, McClay J. Medical and surgical management of subperiosteal orbital abscess secondary to acute sinusitis in children. Int J Pediatr Otorhinolaryngol. 2006;70(11):1853-61.

5. Ozkurt FE, Ozkurt ZG, Gul A, Akdag M, Sengul E, Yilmaz B, et al. Managment of orbital complications of sinusitis. Arq Bras Oftalmol. 2014 Oct;77(5):293-6.

6. Fokkens WJ, Lund VJ, Mullol J, Bachert C, Alobid I, Baroody F, et al. EPOS 2012: European position paper on rhinosinusitis and nasal polyps 2012. A summary for otorhinolaryngologists. Rhinology. 2012 Mar;50(1):1-12.

7. Babar-Craig H, Gupta Y, Lund VJ. British Rhinological Society audit of the role of antibiotics in complications of acute rhinosinusitis: a national prospective audit. Rhinology. 2010 Sep 1;48(3):344-7.

8. Hoxworth JM, Glastonbury CM. Orbital and intracranial complications of acute sinusitis. Neuroimaging Clin N Am. 2010 Nov;20(4):511-26.

9. Jarrett WH, Gutman FA. Ocular complications of infection in the paranasal sinuses. Arch Ophthalmol (Chicago, III 1960). 1969 May;81(5):683-8.

10. Emmett Hurley P, Harris GJ. Subperiosteal Abscess of the Orbit. Ophthalmic Plast Reconstr Surg. 2012;28(1):22-6.

11. Weiss A, Friendly D, Eglin K, Chang M, Gold B. Bacterial periorbital and orbital celIulitis in childhood. Ophthalmology. 1983 Mar;90(3):195-203.

12. Teinzer F, Stammberger $H$, Tomazic P V. Transnasal Endoscopic Treatment of Orbital Complications of Acute Sinusitis: The
Graz Concept. Ann Otol Rhinol Laryngol. 2015;124(5):368-73.

13. Coenraad S, Buwalda J. Surgical or medical management of subperiosteal orbital abscess in children: A critical appraisal of the literature. Rhinology. 2009;47(1):18-23.

14. Siedek V, Kremer a., Betz CS, Tschiesner $\cup$, Berghaus a., Leunig a. Management of orbital complications due to rhinosinusitis. Eur Arch Oto-Rhino-Laryngology. 2010;267(12):1881-6.

15. Starkey CR, Steele RW. Medical management of orbital cellulitis. Pediatr Infect Dis J. 2001 Oct;20(10):1002-5.

16. Garcia GH, Harris GJ. Criteria for nonsurgical management of subperiosteal abscess of the orbit: analysis of outcomes 1988-1998. Ophthalmology. 2000 Aug;107(8):1454-6; discussion 1457-8.

17. Liao JC, Harris GJ. Subperiosteal abscess of the orbit: evolving pathogens and the therapeutic protocol. Ophthalmology. 2015 Mar;122(3):639-47.

18. Harris GJ. Subperiosteal abscess of the orbit. Age as a factor in the bacteriology and 
response to treatment. Ophthalmology 1994 Mar;101(3):585-95.

19. Gavriel H, Yeheskeli E, Aviram E, Yehoshua L, Eviatar E. Dimension of Subperiosteal Orbital Abscess as an Indication for Surgical Management in Children. Otolaryngol -Head Neck Surg. 2011;145(5):823-7.

20. Erickson BP, Lee WW. Orbital Cellulitis and Subperiosteal Abscess: A 5-year Outcomes Analysis. Orbit. 2015 Jun;34(3):115-20

21. Welsh LW, Welsh JJ. Orbital complications of sinus disease. Laryngoscope. 1974 May;84(5):848-56.

22. Harris GJ. Age as a factor in the bacteriology and response to treatment of subperiosteal abscess of the orbit. Trans Am Ophthalmol Soc. 1993 Jan;91:441-516.

23. Brown CL, Graham SM, Griffin MC, Smith RJH, Carter KD, Nerad JA, et al. Pediatric medial subperiosteal orbital abscess: medical management where possible. Am J Rhinol. Jan;18(5):321-7.

24. Liao S, Durand ML, Cunningham MJ. Sinogenic orbital and subperiostea abscesses: microbiology and methicillinresistant Staphylococcus aureus inci- dence. Otolaryngol Head Neck Surg. 2010 Sep;143(3):392-6.

25. Ryan JT, Preciado DA, Bauman N, Pena M Bose S, Zalzal GH, et al. Management of pediatric orbital cellulitis in patients with radiographic findings of subperiosteal abscess. Otolaryngol Head Neck Surg. 2009 Jun;140(6):907-11.

26. Rubin SE, Rubin LG, Zito J, Goldstein MN Eng C. Medical management of orbital subperiosteal abscess in children. J Pediatr Ophthalmol Strabismus. Jan;26(1):21-7.

27. Harris GJ. Subperiosteal abscess of the orbit: older children and adults require aggressive treatment. Ophthal Plast Reconstr Surg. 2001 Nov; 17(6):395-7.

28. Harris GJ. Subperiosteal abscess of the orbit: computed tomography and the clinical course. Ophthal Plast Reconstr Surg. 1996 Mar;12(1):1-8

29. Tabarino F, Elmaleh-Bergès $M$, Quesnel $S$, Lorrot M, Van Den Abbeele T, Teissier N Subperiosteal orbital abscess: volumetric criteria for surgical drainage. Int J Pediatr Otorhinolaryngol. 2015 Feb;79(2):131-5

30. Dewan MA, Meyer DR, Wladis EJ. Orbital cellulitis with subperiosteal abscess: demographics and management outcomes. Ophthal Plast Reconstr Surg. Jan;27(5):330-

Johan Hindkjær Therchilsen Department of Otolaryngology Head \& Neck Surgery Copenhagen University Hospital Copenhagen

Denmark

Tel: +45-2625 0191

E-mail:

johantherchilsen@hotmail.com

ISSN: 2589-5613 / @2019 The Author(s). This work is licensed under a Creative Commons Attribution 4.0 International License. The images or other third party material in this article are included in the article's Creative Commons license, unless indicated otherwise in the credit line; if the material is not included under the Creative Commons license, users will need to obtain permission from the license holder to reproduce the material. To view a copy of this license, visit http://creativecommons.org/ licenses/by/4.0/ 